NOTA DE PESQUISA

\title{
FLEBOTOMÍNEOS (DIPTERA, PSYCHODIDAE) DE ÁREA DE CERRADO NO MUNICÍPIO DE CORUMBATAÍ, CENTRO-LESTE DO ESTADO DE SÃO PAULO, BRASIL
}

\author{
ANDRÉ A. CUTOLO ${ }^{1}$; CLAUDIO J. VON ZUBEN ${ }^{1}$
}

\begin{abstract}
CUTOLO, A.A.; VON ZUBEN, C.J. [Sandflies (Diptera: Psychodidae) from a cerrado area of Corumbataí Municipality, Central East Region of São Paulo State, Brazil]. Flebotomíneos (Diptera, Psychodidae) de área de cerrado no Município de Corumbataí, Centro-Leste do Estado de São Paulo, Brasil. Revista Brasileira de Parasitologia Veterinária, v. 17, n. 1, p.45-49, 2008. Universidade Estadual Paulista, Departamento de Zoologia, Instituto de Biociências, Avenida 24-A, 1515, Bela Vista, Rio Claro, SP 13506-900, Brasil. E-mail: cutoloandre@yahoo.com

Distribution and ecology knowledge of sandfly species is essential for epidemiology vigilance and risk determination for transmission of leishmaniasis. Sandfly trapping was carried out in a cerrado strictu sensu vegetation fragment in a rural area of Corumbataí Municipality, São Paulo State, Brazil, during July to November 2004. Two CDC light traps were used from $18 \mathrm{~h}$ to $8 \mathrm{~h}$, once a month, resulting in 112 total hours of capture. During this period, 60 sandfly specimens of ten different species were sampled. The most abundant species and the one found in all captures was Pintomyia monticola totalizing 15 (25.0\%) specimens, the second more abundant one was P. pessoai with 14 (23.3\%) and the third one was Brumptomyia guimaraesi with 12 (20.0\%) individuals collected. Other captured species were Psathyromyia aragaoi, B. avellari, B. brumpti, B. cunhai, P. bianchigalatiae, Evandromyia termitophila and Nyssomyia whitmani which corresponded to 19 (31.2\%) specimens captured. Pintomyia pessoai and $N$. whitmani presence indicates transmission risk of American Cutaneous Leishmaniasis in the area.
\end{abstract}

KEY WORDS: Cutaneous Leishmaniasis Vector, Sandfly, Cerrado.

\section{RESUMO}

O conhecimento da distribuição e ecologia das espécies de flebotomíneos é fundamental para a vigilância epidemiológica das leishmanioses. A pesquisa e a divulgação do encontro destes insetos colaboram para a determinação do risco de transmissão das mesmas. Realizaram-se capturas de flebotomíneos em fragmento de cerrado strictu sensu em área rural do município de Corumbataí, no período de julho a novembro de 2004. Foram utilizadas duas armadilhas luminosas automáticas do tipo CDC, das $18 \mathrm{~h}$ às $8 \mathrm{~h}$, sendo uma coleta por mês, resultan-

\footnotetext{
${ }^{1}$ Universidade Estadual Paulista (Unesp), Departamento de Zoologia, Instituto de Biociências, Avenida 24-A, 1515, Bela Vista, Rio Claro, SP 13506-900, Brasil. E-mail: cutoloandre@yahoo.com
}

do em 112 horas de exposição. Durante o período de estudo, foi capturado um total de 60 flebotomíneos pertencentes a dez espécies diferentes. A espécie mais abundante e a única presente em todas as capturas foi Pintomyia monticola com um total de 15 (25,0\%) espécimes, a segunda foi P. pessoai com 14 (23,3\%) e a terceira Brumptomyia guimaraesi com 12 (20,0\%) indivíduos coletados. As demais espécies, Psathyromyia aragaoi, B. avellari, B. brumpti, B. cunhai, Pintomyia bianchigalatiae, Evandromyia termitophila e Nyssomyia whitmani somaram 19 (31,2\%) exemplares capturados. A presença de $P$. pessoai e $N$. whitmani indica risco de transmissão da Leishmaniose Tegumentar Americana na área estudada.

PALAVRAS-CHAVE: Vetores de Leishmaniose Tegumentar, fauna, cerrado. 
A Leishmaniose Visceral Americana (LVA) é causada pela Leishmania chagasi e é uma zoonose grave e potencialmente fatal que atinge principalmente crianças desnutridas, sendo transmitida em sua maioria por flebotomíneos do complexo Lutzomyia longipalpis (LAINSON; SHAW, 1978). Inquéritos entomológicos no Estado de São Paulo mostraram a presença desta espécie vetora no ambiente intradomiciliar no município de Araçatuba (COSTA et al., 1997), bem como em outros 44 municípios da região Oeste paulista (CAMARGO-NEVES, 2004) e de outras regiões do Estado de São Paulo (FORATTINI et al., 1970; CUTOLO et al., 2006).

No Estado de São Paulo foram identificados de 1999 a outubro de 2007, pela Secretaria Estadual de Saúde, através do Centro de Vigilância Epidemiológica, casos humanos de Leishmaniose Visceral Americana em 43 municípios, prevalecendo os do Oeste paulista, num total de 1006 casos, sendo 91 fatais. Nesta região foram ainda avaliados por sorologia 267.383 cães de 1999 até 2004, sendo 18.513 $(6,92 \%)$ considerados positivos para a infecção (CENTRO DE VIGILÂNCIA EPIDEMIOLÓGICA, 2007).

A Leishmaniose Tegumentar Americana (LTA) é conhecida no Estado de São Paulo há mais de um século, quando era conhecida pelo seu caráter ocupacional, acometendo trabalhadores associados a atividades de desmatamento, sendo uma zoonose silvestre e o homem hospedeiro acidental do protozoário (TOLEZANO, 1994). O número de casos passou a decrescer no terço final de 1950, quando a área florestada do Estado havia caído a níveis próximos a $18 \%$ da cobertura original. Apesar disso, de forma surpreendente a incidência da doença voltou a crescer por volta de 1978 na região sul do Estado, e daí em diante a incidência mantém-se crescente e ocorre de forma endêmica em áreas de colonização estabelecida, sem caráter ocupacional, com ciclo de transmissão zoonótico associado a animais domésticos ou sinantrópicos (TOLEZANO, 1994).

A LTA é considerada importante tanto pela sua extensa distribuição geográfica no Brasil como pelas graves lesões cutâneas e mucocutâneas que provoca, com envolvimento psicológico do doente e reflexos no campo social e econômico (MINISTÉRIO DA SAÚDE, 1994). É causada por diferentes espécies do gênero Leishmania, sendo Leishmania braziliensis o agente principal para o Estado de São Paulo (GOMES, 1994). Os principais transmissores são flebotomíneos do complexo Nyssomyia intermedia ( $N$. intermedia no litoral e $N$. neivai no interior), associadas ainda à $N$. whitmani e Migonemyia migonei como possíveis espécies vetoras secundárias (CAMARGO-NEVES et al., 2002). No Estado de São Paulo, de 1996 a início de 2007, foram relatados 6466 casos autóctones humanos da doença (CVE, 2007). Embora não exista transmissão de LVA na região, no município de Rio Claro, vizinho à Corumbataí, no período de 2000 a setembro de 2007 foram diagnosticados 33 casos humanos de LTA (CVE, 2007).

O conhecimento da distribuição e ecologia das espécies de flebotomíneos vetoras é fundamental para a vigilância epidemiológica das leishmanioses. O encontro de $L u$. longipalpis em área rural de Itirapina (CUTOLO et al., 2006), município sem transmissão de LVA e também vizinho à Corumbataí, motivou a realização da pesquisa para se determinar os riscos do estabelecimento da LVA na região e de transmissão da LTA no local.

Foi considerado um fragmento de cerrado nativo em área rural do município de Corumbataí, SP (entre longitudes $47^{\circ} 40^{\prime}$ e $47^{\circ} 45^{\prime}$ O e latitudes $22^{\circ} 10^{\prime}$ e $22^{\circ} 15^{\prime}$ ' S e entre 850 e 790 m de altitude), situado a $33 \mathrm{~km}$ da cidade de Rio Claro. A área de 38,7 ha. está protegida de cortes e queimadas desde 1962 e é uma reserva pertencente à FAPESP, sob responsabilidade do Instituto de Biociências da UNESP de Rio Claro, SP. A vegetação varia entre campo limpo, capoeira, cerrado propriamente dito e cerradão. Possui espécies arbóreas de 4 a $12 \mathrm{~m}$ de altura, representantes das famílias Myrtaceae, Thymelaceae, Lauraceae, Vochysiciaceae e Melastomaceae. O solo sob a vegetação foi classificado como Latossolo Vermelho Amarelo de textura média. O clima é caracterizado como tropical úmido, compreendendo uma estação chuvosa de outubro a março e outra seca, de abril a setembro (ATTILI, 1989). As armadilhas foram posicionadas no centro do fragmento, na área de cerrado propriamente dito, vegetação dominante na área (Figura 1).

Duas armadilhas luminosas automáticas do tipo CDC foram posicionadas a uma distância de 30 a $50 \mathrm{~cm}$ do solo, cerca de 50 metros entre si. Funcionaram por um período ininterrupto das 18 às 8 horas do dia seguinte, nos dias 31 de julho, 28 de agosto, 25 de setembro e 13 de novembro de 2004, resultando em 112 horas de exposição. Os insetos capturados foram mortos por congelamento, clarificados, corados e montados em lâmina de vidro de acordo com Marcondes (1998) e classificados de acordo com Galati (2003). Os exemplares capturados encontram-se depositados na coleção de invertebrados do Departamento de Zoologia, Instituto de Biociências da UNESP, Campus de Rio Claro.

Houve diferença na quantidade de exemplares capturados nas três primeiras coletas comparado à última coleta. A coleta de julho rendeu apenas um exemplar, a de agosto cinco e a de setembro 13 indivíduos, dando um total de 19 exemplares, correspondendo a 31,77\% do total capturado. A coleta realizada em outubro proporcionou a captura de 41 exemplares, 68,33\% do total capturado durante o estudo. Tal aumento na quantidade de insetos capturados se deve provavelmente à maior umidade devido ao início do período das chuvas. Segundo Forattini (1973) a umidade é aparentemente o fator microclimático mais importante na sobrevivência de flebotomíneos e o aumento da mesma nos 

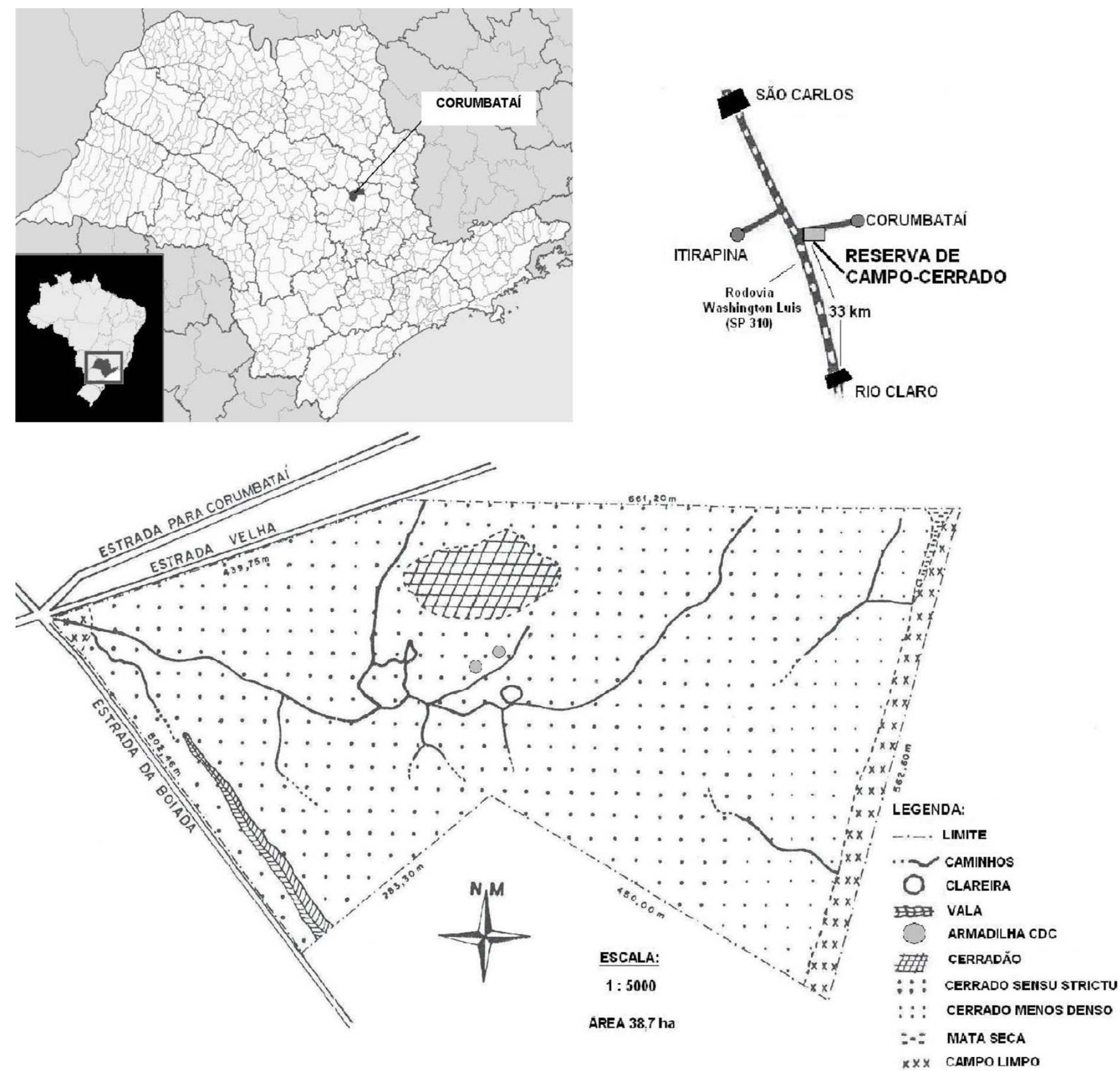

Figura 1. Localização geográfica da reserva de campo-cerrado e da área de estudo no município de Corumbataí, SP.

abrigos e criadouros promoveria a emergência dos insetos adultos.

Na Tabela 1, observa-se que dos 60 insetos capturados, $22(36,67 \%)$ pertencem a quatro espécies distintas de flebotomíneos do gênero Brumptomyia. Os flebotomíneos desse gênero não têm importância epidemiológica na transmissão das leishmanioses, pois têm hábitos zoofílicos restritos, realizando repasto sangüíneo em tatus (Dasipodidae: Edentata) (FORATTINI, 1973) o que é um indicativo da presença desses mamíferos na área. Pintomyia monticola espécie antropofílica, dominante e presente em todos os meses de captura é suspeita de ser transmissora de Leishmania enrietti (LAINSON, 1997).

A ausência de $N$. neivai nas capturas confirma a preservação da mata estudada, já que esta espécie foi dominante em estudos realizados em áreas rurais alteradas habitadas pelo homem nos municípios vizinhos de Rio Claro (CUTOLO, 2003) e Ipeúna (CUTOLO et al., 2006). No período trabalhado a espécie $L u$. longipalpis não foi notificada no local.

Das demais espécies capturadas, apenas $N$. whitmani (HOCH et al.,1986) e P. pessoai (FORATTINI et al., 1972) 
Tabela 1. Total de flebotomíneos, por espécie e sexo, capturados com armadilhas CDC's em área de Cerrado, no período de Julho a Novembro de 2004 em Corumbataí, SP.

\begin{tabular}{lcccc}
\hline \multirow{2}{*}{ Espécie } & \multicolumn{4}{c}{ Número de Flebotomíneos } \\
\cline { 2 - 5 } & Machos & Fêmeas & Total & $\%$ \\
\hline Pintomyia monticola & 2 & 13 & 15 & 25,00 \\
P. pessoai & 7 & 7 & 14 & 23,33 \\
Brumptomyia guimaraesi & 9 & 3 & 12 & 20,00 \\
Psathyromyia aragaoi & 2 & 4 & 6 & 10,00 \\
Brumptomyia spp. & - & 4 & 4 & 6,67 \\
B. avellari & 1 & 2 & 3 & 5,00 \\
B. brumpti & - & 2 & 2 & 3,33 \\
Evandromyia termitophila & - & 1 & 1 & 1,67 \\
Nyssomyia whitmani & - & 1 & 1 & 1,67 \\
P. bianchigalatiae & - & 1 & 1 & 1,67 \\
B. cunhai & 1 & - & 1 & 1,67 \\
\hline Total & 22 & 38 & 60 & 100,00 \\
\hline
\end{tabular}

são consideradas espécies importantes na transmissão da $L$. braziliensis, agente etiológico da LTA. Como as áreas de vegetação preservadas são cada vez mais raras no território paulista, tais espécies vêm perdendo importância na epidemiologia da LTA, ficando o papel principal de transmissão no Estado e em todo o Sudeste brasileiro para Nyssomyia intermedia s.l., espécie mais bem adaptada às áreas alteradas pelo homem (GOMES, 1994; CAMARGO-NEVES et al., 2002). A presença de $P$. pessoai e $N$. whitmani na área indica risco de transmissão da Leishmaniose Tegumentar Americana.

Agradecimento:- À Prof. Dra. Eunice Aparecida Biachi Galati pelo auxílio na identificação de espécimes capturados.

\section{REFERÊNCIAS BIBLIOGRÁFICAS}

ATTILI, D.S. Sucessão fúngica e decomposição da fração foliar da serrapilheira de cerrado no município de Corumbataí, SP. 1989. 43f. Dissertação (Mestrado) Universidade Estadual Paulista - UNESP, Rio Claro, 1989.

CAMARGO-NEVES, V.L.F.; GOMES, A.C.; ANTUNES, J.L.F. Correlação da presença de espécies de flebotomíneos (Diptera: Psychodidae) com registros de casos de leishmaniose tegumentar americana no Estado de São Paulo, Brasil. Revista da Sociedade Brasileira de Medicina Tropical, v.35, n.4, p.299-306, 2002.

CAMARGO-NEVES, V.L.F. A Leishmaniose Visceral Americana no Estado de São Paulo: Situação Atual. Boletim Epidemiológico Paulista, ano 1, n. 6, 2004. Disponível em: <http:॥www.sucen.sp.gov.br>. Acesso em: 25 set. 2005

COSTA, A.I.P.; CASANOVA, C.; RODAS, L.A.C.; GALATI, E.A.B. Atualização da distribuição geográfica e primeiro encontro de Lutzomyia longipalpis em área urbana no Estado de São Paulo, Brasil. Revista de Saúde Pública. v. 31, n. 6, p.632-633, 1997.

CUTOLO, A.A. Inquérito entomofaunístico de flebotomíneos (Diptera: Psychodidae) e sorologia canina para deteç̧ão de anticorpos anti-Leishmania, no município de Rio Claro, São Paulo. 2003. 101 f. Dissertação (Mestrado) - Universidade Estadual Paulista UNESP, Rio Claro, 2003.

CUTOLO, A.A.; CAMARGO, D.A.; CUTOLO, A.A.; VON ZUBEN, C.J.; GALATI, E.A.B. Lutzomyia longipalpis (Diptera, Psychodidae) em Cuesta Basáltica na bacia hidrográfica do Rio Corumbataí, Região Centro-leste do Estado de São Paulo. In: CONGRESSO BRASILEIRO DE PARASITOLOGIA VETERINÁRIA, 14, 2006, Ribeirão Preto. Anais... Ribeirão Preto: CBPV, 2006. p.195.

CENTRO DE VIGILÂNCIA EPIDEMIOLÓGICA “Prof. Alexandre Vranjac", Divisão de Zoonoses, Secretaria de Estado da Saúde. Leishmaniose Visceral Americana Humana. Casos Autóctones e Óbitos de LVA, no Estado de São Paulo, 1999 a 2007. Disponível em: $<$ http://www.cve.saude.sp.gov.br>. Acesso em: 15 nov. 2007.

FORATTINI, O.P.; PATTOLI, D.B.G.; RABELLO, E.X.; FERREIRA, O.A. Infecção natural de flebotomíneos em foco enzoótico de leishmaniose tegumentar no Estado de São Paulo, Brasil. Revista de Saúde Púbica, v. 6, n. 4, p. 431-433, 1972.

FORATTINI, O.P.; RABELLO, E.X.; PATTOLI, D.G.B. Sobre o encontro de Lutzomyia longipalpis (Lutz; Neiva, 1912) no Estado de São Paulo, Brasil. Revista de Saúde Pública. v. 4, n. 1, p. 99-100, 1970.

FORATTINI, O.P. Entomologia Médica. São Paulo: Edgard Blücher, 1973. 658 p., v. 4.

GALATI, E.A.B. Morfologia, terminologia de adultos e identificação dos táxons da América. In: RANGEL E.F.; LAINSON R. Flebotomíneos do Brasil, Rio de Janeiro: Fiocruz, 2003. p. 53-175.

GOMES, A.C. Sand fly vectorial ecology in the State of São Paulo. Memórias do Instituto Oswaldo Cruz, v. 89, n. 3, p. 457-60, 1994.

HOCH, A.; RYAN, L.; VEXENAT, J.A.; ROSA, A.C.O.C.; BARRETTO, A.C. Isolation of Leishmania braziliensis braziliensis and other trypanosomatids from Phlebotomine in a mucocutaneus leishmaniasis area, Bahia, Brazil. Memórias do Instituto Oswaldo Cruz, v. 81, Supl., p. 62, 1986.

LAINSON, R. On Leishmania enriettii and other enigmatic Leishmania species of the neotropics. Memórias do Instituto Oswaldo Cruz, v. 92, n. 3, p. 377-387, 1997.

LAINSON, R.; SHAW, J.J. Epidemiology and ecology of leishmaniasis in Latin-America. Nature. v. 273, Supl., p. 595-600, 1978. 
MARCONDES, C.B. An improved technique for the dissection of female genitalia of Phlebotominae sandflies (Diptera: Psychodidae), with an improvement in the handling of insects. Memórias do Instituto Oswaldo Cruz, v. 93, n. 1, p. 109, 1998.

MINISTÉRIO DA SAÚDE. Guia de Controle da Leishmaniose Tegumentar Americana. Brasília: Funda- ção Nacional de Saúde, Centro Nacional de Epidemiologia, Coordenação Nacional de Dermatologia Sanitária. 1994. 44p.

TOLEZANO, J.E., Ecoepidemiological aspects of american cutaneous leishmaniasis in the State of São Paulo, Brazil. Memórias do Instituto Oswaldo Cruz, v. 89, n.3, p. 427434, 1994.

Recebido em 13 de dezembro de 2006.

Aceito para publicação em 14 de março de 2007. 